\title{
Médiévales
}

Langues, Textes, Histoire

44 | printemps 2003

Le diable en procès

\section{Quelques hypothèses à propos de l'artiste roman}

\section{Pierre Alain Mariaux}

\section{OpenEdition}

\section{Journals}

Édition électronique

URL : https://journals.openedition.org/medievales/741

DOI : 10.4000/medievales.741

ISSN : 1777-5892

\section{Éditeur}

Presses universitaires de Vincennes

\section{Édition imprimée}

Date de publication : 1 juin 2003

Pagination : 199-214

ISBN : 2-84292-142-9

ISSN : 0751-2708

\section{Référence électronique}

Pierre Alain Mariaux, "Quelques hypothèses à propos de l'artiste roman », Médiévales [En ligne], 44 | printemps 2003, mis en ligne le 26 octobre 2005, consulté le 23 avril 2022. URL : http://

journals.openedition.org/medievales/741 ; DOI : https://doi.org/10.4000/medievales.741

Ce document a été généré automatiquement le 23 avril 2022

Tous droits réservés 


\title{
Quelques hypothèses à propos de l'artiste roman
}

\author{
Pierre Alain Mariaux
}

1 Le travail de l'historien de l'art suppose la maitrise des outils propres à sa discipline, ce que, pour simplifier, on nommera l'iconographie et la stylistique. Mais on attend de lui, à plus forte raison lorsqu'il aborde le Moyen Âge, qu'il manifeste aussi une évidente familiarité avec les méthodes et les résultats des disciplines sœurs que sont la théologie, le droit canon, la liturgie, l'hagiographie, la littérature, la paléographie, l'histoire, l'archéologie, etc., qui l'aideront à situer l'objet dans son contexte et favoriseront par conséquent une analyse plus précise. Ceci explique probablement la multiplication des études monographiques, au détriment des synthèses qui ne trouveraient leur voie que dans des survols généraux n'élevant guère les débats. Les deux formes d'études présentent pourtant chacune leurs défauts: la monographie semble parfois se perdre dans les détails les plus infimes, que la synthèse abandonne très souvent sur le côté.

2 Deux ouvrages parus récemment ont toutefois la prétention d'échapper à ces écueils : le premier est consacré au programme sculpté de la cathédrale d'Autun ${ }^{1}$, le second, bien que son auteur s'en défende, est une synthèse sur l'image romane ( $\mathrm{du} \mathrm{IX}^{\mathrm{e}}$ au milieu $\mathrm{du} \mathrm{XII}^{\mathrm{e}}$ siècle $)^{2}$. Leur lecture est stimulante à plus d'un titre et soulève de nombreuses questions. Je ne souhaite pas présenter les arguments des deux auteurs dans le détail, mais m'intéresser aux seules réflexions concernant l'artiste. Les études pionnières de P. C. Claussen, A. Legner, et P. Skubiszewski ont patiemment contribué à changer notre vision de l'artiste médiéval', et l'on doit aujourd'hui fortement relativiser l'image traditionnelle que donnaient V. Egbert ou A. Martindale ${ }^{4}$, à savoir celle d'un artisan écrasé par le travail, exécutant les volontés de son commanditaire, borné par l'horizon de son travail «mécanique ». Les deux ouvrages recensés n'épuisent certainement pas la richesse du problème mais, par leur approche neuve, ils indiquent des voies de recherche fructueuses. J'en relève quelques-unes dans les pages suivantes, qui m'aideront à formuler quelques hypothèses sur l'image de l'artiste médiéval.

«Legends in Limestone » 
3 Le touriste contemporain se rend à Autun pour y admirer «l'œuvre d'un artiste exceptionnel »: le tympan du Jugement dernier, sculpté par Gislebertus. Mais en approchant la cathédrale, le pèlerin médiéval aurait-il spontanément associé la trace écrite (GISLEBERTUS), à supposer qu'il ait pu la voir et la lire, au sculpteur du tympan comme nous le faisons aujourd'hui ? Telle est la question initiale que pose Linda Seidel, car ce nom gravé au linteau condense toutes les attentes et conditionne l'étude du décor sculpté de Saint-Lazare. Dès sa (re)découverte vers 1830, soutient l'auteur, l'intérêt s'est déplacé de l'ensemble architectural à la personnalité du sculpteur et à son destin de "grand homme ». À Autun en d'autres termes, Gislebertus évince Lazare pour en devenir le héros et comme le nouveau saint. L'auteur montre comment l'on forge, maintenant et alors, l'identité de Gislebertus, tandis que l'on inscrit la construction de Saint-Lazare dans la mémoire locale. En premier lieu, elle cherche à déterminer quelle signification le Moyen Âge accorde à la commémoration à travers l'usage du nom. Le nom est une orthographic fingerprint, dit Linda Seidel, qui permet d'identifier l'ancêtre comme le descendant; plus particulièrement, le fait de nommer établit l'histoire dans le futur comme dans le passé (p. 14). Pour l'auteur, l'insertion du nom au sein de l'épigraphe participe dès lors d'une stratégie éponyme, dont la fonction est de commémorer et de magnifier l'action particulière d'un ancêtre, un phénomène avéré à la même époque non seulement en Bourgogne, mais dans toute l'Europe (p. 15). Le nom associe ainsi à Saint-Lazare un individu nommé Gislebertus et en perpétue le souvenir. Comme elle l'affirmera plus avant dans l'analyse, Linda Seidel ne pense pas qu'il puisse s'agir du sculpteur - elle qualifie Gislebertus de fictitious fabricator (p. 52) -, mais de quelque important personnage engagé dans l'invention des reliques de Lazare, un moral mover en quelque sorte (p. 72). En parcourant les chartes contemporaines de la construction de Saint-Lazare, elle découvre ainsi deux Gislebertus à Autun: un capellanus, cité comme témoin dans un acte de donation en faveur de la cathédrale Saint-Nazaire, datable du deuxième quart du XII ${ }^{\mathrm{e}}$ siècle ; un buticularius, témoin en 1120 de diverses transactions entre le duc de Bourgogne et l'évêque d'Autun d'une part, entre le roi Louis VI et les églises de Sens et d'Avallon d'autre part. À cette époque, le nom est somme toute assez commun à Autun et dans les alentours, si bien que l'on peut se demander avec l'auteur si ce n'est pas l'un ou l'autre de ces Gislebertus qui serait célébré au tympan. En 1120 en effet, le second nommé intervient comme témoin lors de la rétrocession à l'évêque d'Autun de l'église d'Avallon, dédiée à Marthe et Lazare, et c'est à peu près au même moment que l'évêque décide la construction d'un nouvel écrin pour accueillir les reliques de Lazare (p. 59).

Dans son entreprise d'identification pourtant, Linda Seidel écarte rapidement ce Gislebertus buticularius - «[...] would he have been important enough to merit such a prominently situated inscription on a new church in one of Burgundy's most important cities?»(p.15) - au profit d'un troisième Gislebertus, comte d'Autun puis duc de Bourgogne ( ca 915-956; voir le tableau généalogique donné en p.66-67), à qui l'on attribue un rôle important dans l'acquisition des précieuses reliques. C'est cet ancêtre distant de la famille ducale, affirme l'auteur, qui se trouve désormais immortalisé au tympan d'un reliquaire qu'il a contribué à construire, même de son lointain passé, et non pas "l'un des plus grands artistes du Moyen Âge », conjecture romantique dit Linda Seidel, hypothèse fondée sur une conception anachronique de la personnalité artistique médiévale (p.15-16). Le Christ dans sa mandorle, dont le relief assez plat évoque un sceau épiscopal, scelle la charte lapidaire (stone charter) figurée par le tympan. Dès lors, Gislebertus apparaît comme l'auteur et le garant d'une transaction et, 
bien qu'elle ne le dise pas en ces termes, l'insertion de GISLEBERTUS HOC FECIT au cœur de l'épigraphe s'apparente au signum du témoin. L'usage de l'expression «charte lapidaire », déjà qualifié d'impropre et de peu satisfaisant par Robert Favreau ${ }^{6}$, résume cependant parfaitement la pensée de l'auteur. Définie peut-être trop grossièrement par "words inscribed on buildings" (p.15) pour devenir en fin d'analyse une "giant pancarte» (p.159), l'expression synthétise le travail de réécriture et de souvenir accompli par les constructeurs de Saint-Lazare, conçu par eux comme un document d'archive. Toutefois, cette archive de pierre ne se limite pas au nom seul, mais elle est constituée par l'édifice dans son entier. De même que l'on construit la légende ou réarrange les faits de mémoire à mesure que l'on édifie, de même le spectateur (sans doute aussi le lecteur) en actualise le sens. Pour une audience locale, Saint-Lazare sert dès lors à mettre en relation une histoire, que l'on veut croire oubliée, et la mémoire vivante: l'église est à la fois un acte de construction et un acte d'imagination. L'inscription du nom dans l'épigraphe sert ainsi à créer un lien, une continuité entre le moment présent et les événements du passé. Si Gislebertus doit être considéré comme un moral mover plutôt qu'un artistic maker, et s'il est honoré publiquement comme « auteur » de l'œuvre, c'est, soutient Linda Seidel, dans le but de donner à l'édifice une apparence vénérable en en distinguant la noble origine (p. 72).

5 L'incise GISLEBERTUS HOC FECIT dans l'épigraphe doit donc être lue comme un tribut à et un témoignage de la donation ducale, l'événement légendaire qui a servi de déclencheur à toute l'entreprise. Le travail de mémoire sur les origines motive ainsi la nouvelle construction d'Autun et se développe en parallèle avec l'édification, pour se fondre bientôt avec elle. Le procédé ne mène pas à l'élaboration d'un texte: on ne réécrit pas à proprement parler le passé, mais on édifie un monument comme s'il s'agissait d'un texte, d'une stone charter; en d'autres termes, on inscrit le présent dans le passé. La construction de Saint-Lazare se substitue au témoignage de la vie du saint et l'incorporation d'un nom au cœur de la narration matérielle le transmet à la mémoire comme patron putatif, de la même façon qu'une charte porte crédit à un fondateur. Autun devient ainsi une machine à construire des fictions. Le décor sculpté rend légitime mais surtout efficace la prétention épiscopale selon laquelle, par le passé, un comte carolingien, Gislebertus, fit quelque chose pour cette église. À Saint-Lazare, le tympan agit en effet comme une pancarte, à savoir un résumé d'actes antérieurs (p. 159), qui défend désormais aux descendants du comte de remettre en cause la donation originale inscrite au tympan ou de douter de son authenticité. S'ils essayaient toutefois, ils endureraient les peines éternelles des damnés figurés au tympan jusqu'à leur résurrection, cette même résurrection dont Lazare est un témoin, autrefois par son propre exemple, aujourd'hui par sa présence physique à Autun.

6 En écartant d'emblée l'opinion commune selon laquelle Gislebertus ne peut être que le sculpteur d'Autun, Linda Seidel parvient à montrer que la construction de Saint-Lazare est aussi une construction posée dans l'histoire. En ce sens, il se peut que le Gislebertus célébré dans l'épigraphe soit le comte d'Autun, à qui l'on attribue tout le mérite de l'apparition des reliques de Lazare. Mais lorsque Linda Seidel rapproche la «signature » de Gislebertus du portrait de Suger au tympan de Saint-Denis, tous deux placés aux pieds d'un Christ en gloire, elle fait cependant la part belle au commanditaire ou au donateur, au détriment de l'artiste. Car il est tout autant légitime d'imaginer qu'un sculpteur agisse de la même façon et qu'il soit lui aussi engagé dans un long processus $\mathrm{du}$ souvenir (des indices existent, comme on le verra plus bas, qui permettent d'envisager l'hypothèse). Dès lors, en modifiant une formule consacrée (hoc fecit à la 
place de l'usuel me fecit), le sculpteur Gislebertus montrerait, une fois le travail accompli, une fierté légitime, justifiée par l'ampleur de la tâche : créer un monument qui donne l'impression d'avoir déjà une histoire, pour répondre aux vœux de l'évêque et de sa communauté. On ne peut donc pas exclure la participation d'un sculpteur à une telle entreprise. Or, si Linda Seidel a sans doute raison d'affirmer que « no other large tympanum is "signed" in this way " (p.12), et même si cela revient à évacuer d'emblée l'hypothèse selon laquelle Gislebertus est malgré tout un sculpteur, elle néglige toutefois les exemples comparables qui pourraient éclairer le cas d'Autun. Avant même de songer à rattacher un individu au nom qui s'offre au regard, il serait sans doute nécessaire de situer l'épigraphe dans le contexte approprié, en l'occurrence les « signatures » d'artistes des $\mathrm{XI}^{\mathrm{e}}$ et XII ${ }^{\mathrm{e}}$ siècles. À ce sujet, l'excellent outil qu'est le Corpus des Inscriptions de la France Médiévale ne semble pas assez mis à contribution. Deux critères jouent un rôle essentiel : la formule utilisée et la localisation de la subscriptio.

Signatures d'artistes

7 L'épigraphe qui court au linteau se compose de quatre hexamètres léonins riches, interrompue en son centre sous les pieds du Christ par une "incise »: GISLEBERTUS HOC FECIT. La subscriptio vient s'intercaler entre une promesse de résurrection pour les justes et la vision des peines éternelles promises aux impies (la « signature » doit sans doute être lue comme si elle était placée au-dessus du reste de l'inscription et non pas la couper, selon un procédé fréquent au Moyen Âge). Malgré sa présence centrale, Gislebertus ne cherche ni à se poser en juge, ni à se substituer au Christ; l'inscription sur la mandorle l'en dispense : omnia dispono solus, dit le Christ. Par contre, quelqu'un, qui n'est pas l'œuvre elle-même, parle à la place de Gislebertus pour affirmer qu'il a fait cela. À suivre le Corpus, l'usage de la formule $X$ hoc fecit (ou X fecit hoc) n'est pas attesté pour les sculpteurs dans la France du $\mathrm{XI}^{\mathrm{e}}$ et $\mathrm{du} \mathrm{XII}^{\mathrm{e}}$ siècle. Pour trouver des exemples comparables à Autun, il faut sortir des frontières.

$8 \mathrm{Au}$ début du xI ${ }^{\mathrm{e}}$ siècle, Bernward de Hildesheim signe de la sorte un crucifix d'argent et un plat de reliure (BERNWARDUS PRESUL HOC FECIT). Au monastère de San Salvador à Nogal de las Huertas (province de Palencia), une épigraphe du XII-XIII ${ }^{\mathrm{e}}$ siècle commémore la comtesse Elvira Sánchez dans les mêmes termes (ELVIRA SANSES HOC FECIT), tandis que l'on conserve la signature du sculpteur (XEMENUS FECIT ET SCULPSIT ISTAM PORTICUM. ORATE PRO ЕO). Sur le panneau de droite de la porte de bronze de l'abbaye du Mont-Cassin, datée de 1066, une inscription précise Hoc FECIT MAURO FILIUS PANTALEONIS. Si l'exemple espagnol ne pose pas de problème, les avis sont partagés quant à savoir si l'évêque d'Hildesheim était effectivement un orfèvre, comme le soutient Thangmar dans sa Vita Bernwardi. À mon avis, il n'existe aucune raison objective de refuser à Bernward des occupations artistiques, et l'on doit croire Thangmar. Il est peu probable enfin que Mauro soit l'artiste : il suit plus vraisemblablement les pas de son père Pantaléon, un marchand d'Amalfi qui a offert des portes similaires à la cathédrale de sa cité et au Monte Sant'Angelo. Ces trois premiers exemples montrent que la même formule ( $X$ hoc fecit) peut servir à commémorer un artiste, un commanditaire et un donateur.

9 On s'accorde à reconnaître les signatures de magister Petrus au tympan de l'abbaye de Sant'Eutizio (près de Norcia, vers 1190) et de Bernard de Tréviers au linteau de SaintPierre de Maguelonne (1178), qui reproduisent la formule, comme celles des deux graveurs de l'épigraphe et non des sculpteurs. Il existe bon nombre de signatures, en Italie surtout, qui reprennent la formule $X$ hoc fecit, mais les signataires accompagnent toujours leur nom d'un titre, précisent ce qu'ils ont fait ou qualifient leur travail. Par 
conséquent, il est pratiquement impossible de les confondre avec le commanditaire. En France à la même époque, si l'artiste signe, il utilise le plus souvent la formule $X$ me fecit. Et si l'on retrouve les signatures sur toutes sortes d'objets - ustensiles liturgiques, mobilier d'église, éléments architectoniques tels que chapiteaux ou voussoirs -, il semble pourtant que les sculpteurs préfèrent les chapiteaux. Quelques exemples présentent toutefois des signatures au tympan, mais le plus souvent l'artiste signe sur l'un des côtés du linteau. Ainsi à Carennac, vers 1100, la signature court sur les tailloirs des deux chapiteaux situés à gauche de la porte: GIRBERTUS CEMENTARIUS FECIT ISTUM PORTARIUM. BENEDICTA SIT ANIMA EIUS. On retrouve la signature d'un second GIRBERTUS au linteau du portail central de l'église Saint-Pierre à Champagne, daté généralement du troisième quart du XII ${ }^{\mathrm{e}}$ siècle. Au tympan occidental de l'ancienne collégiale de SainteEngrâce, daté de la seconde moitié du XII siècle et décoré d'un chrisme soutenu par deux anges, la signature BERNARDUS ME FECIT figure à la suite de l'inscription qui court autour du monogramme ${ }^{7}$. À Saint-Pourçain-sur-Sioule, on conserve un me fecit esseulé. Au tympan de Saint-Ursin de Bourges, daté du second quart du XII ${ }^{e}$ siècle ${ }^{8}$, un cartouche placé au centre du linteau accueille une subscriptio qui laisse cependant peu de doute : GIRAULDUS FECIT ISTAS PORTAS. Le tympan occidental de l'église d'Ameugny, daté de la seconde moitié du XII siècle, est décoré d'une croix inscrite dans un cercle. Une longue épigraphe court autour, qui conserve les noms du commanditaire et de l'artiste : + AVE MARIA GRATIA PLENA DOMINUS TECUM IOHANNES CAPELLANUS TASIACO ATQUE AMUNIACI SCRIPSIT HOC ET SEgUinUS LAPIFEX MELEI. Comme à Maguelonne ou à Sant'Eutizio, le hoc désigne l'épigraphe elle-même, tandis que Seguinus doit préciser son état de tailleur de pierre (lapifex), pour se distinguer du prêtre Jean sans doute. On pourrait multiplier les exemples encore : les tympans d'Ydes, de Tredos, de Lavedan, d'Ardentes, de Til-Châtel, etc., présentent également la signature d'un sculpteur. De toute manière, lorsque l'artiste choisit de signer au tympan, la signature répète la formule $X$ me fecit ou désigne explicitement ce qu'il a fait.

10 Sur la base de ces exemples, nous serions donc tenté de dire avec Linda Seidel que Gislebertus n'est pas le sculpteur d'Autun. Pourtant, on attendrait d'un duc qu'il manifeste son état dans l'épigraphe. S'il est vrai que la comtesse Elvira Sánchez ne précise pas non plus son titre, c'est sans doute parce qu'elle se place sous l'autorité du roi Ferdinand, également mentionné dans l'inscription commémorative du monastère de Nogal de las Huertas. Dès lors, décider de la fonction de Gislebertus sur la seule base de l'épigraphe s'apparente plutôt à un acte de foi. Car s'il n'existe pas d'exemple français que l'on pourrait utilement comparer à Autun, les exemples rappelés ci-dessus montrent que la formule hoc fecit désigne l'artiste comme le donateur ou le commanditaire. En certaines inscriptions par ailleurs, le sens factitif du verbe facere ressort sans peine, mais cela ne me paraît pas être le cas ici. Si l'on confronte Autun aux exemples d'Ameugny et de Maguelonne, la comparaison semble indiquer que Gislebertus a composé l'épigraphe. En ce sens, rien ne s'oppose à ce qu'il soit aussi un sculpteur, et l'on peut envisager dans la foulée l'hypothèse selon laquelle il s'agit du buticularius mentionné dans les chartes autour de 1120 .

11 Ce qui pose également problème au tympan d'Autun, et Linda Seidel ne soulève pas ce point, c'est l'utilisation du pronom hoc, objet d'une section des Institutiones grammaticae de Priscien, un traité largement copié aux $\mathrm{XI}^{\mathrm{e}}$ et $\mathrm{XII}^{\mathrm{e}}$ siècles. La question du pronom devient réellement délicate si l'on s'attache à la confection du corps du Christ à l'autel, un problème qui préoccupe aussi les artistes me semble-t-il. Sans aller trop avant dans 
cette direction, et sans en épuiser faute de temps toute la richesse, je remarque que ce problème est posé par Bérenger de Tours dans sa définition du sacrement comme signe. Toute affirmation ne pouvant subsister si l'on en supprime l'un des termes, dit-il, le sujet est pris au sens propre (proprie) tandis que le prédicat l'est au sens figuré (tropice), sans quoi l'affirmation n'est logiquement pas possible ${ }^{9}$. Et ceci est d'autant plus vrai de l'énoncé « Hoc est corpus meum ». Pour appuyer sa démonstration, Bérenger use d'une image : «Christus est angularis lapis ». Comme il est évident que le Christ n'est pas une pierre, l'énoncé doit s'entendre au sens figuré. L'emprunt de cette métaphore architecturale ne se fait certes pas par hasard et je me demande si, par l'usage du pronom hoc, les artistes ne jouent pas également sur cette ambiguïté : le pronom désigne certes quelque chose que l'on a sous les yeux, mais signifie quelque chose d'autre. Ainsi, dans le cas de l'épigraphe d'Autun, comme dans celui de la subscriptio du sculpteur Anselme à Milan (porta romana datée de 1171 : HOC OPUS ANSELMUS FORMAVIT [...]) par exemple, le pronom renvoie au «double spirituel » de l'œuvre que l'on a sous les yeux : l'église spirituelle, la Jérusalem céleste, la porte du Paradis, etc. Les artistes du $\mathrm{XII}^{\mathrm{e}}$ siècle me semblent pleinement conscients du phénomène. D'une part, comme on le verra par la suite avec l'exemple d'Autry-Issards, ils sont capables d'inscrire ouvertement leur œuvre dans cette problématique. D'autre part, en traçant un tableau de la fréquence des signatures d'artistes, j'ai pu constater que leur nombre croît de manière régulière depuis $\mathrm{le} \mathrm{Vl}^{\mathrm{e}}$ siècle pour connaître, autour de 1075 environ, une soudaine explosion. Il semble en effet que la fin du XI siècle soit le théâtre d'une soudaine prise de conscience identitaire de l'artiste. Je formulerai l'hypothèse générale selon laquelle la fréquence des signatures est l'indice de l'existence d'une relation entre « réforme grégorienne » et «naissance de l'individu», dont profitent les artistes. On sait que la réforme grégorienne consacre la fonction sacerdotale. Or, je soupçonne qu'une analyse serrée de la figure du sacerdos peut être décisive pour comprendre l'image de l'artiste médiéval. C'est entre autres par rapport à l'Incarnation que se situe l'artiste lorsqu'il « crée » ou « fait » quelque chose en effet. Les signatures de Rotbertus à Notre-Dame-du-Port ou de Gofridus à Saint-Pierre de Chauvigny par exemple, sont placées de telle sorte qu'elles posent une relation entre Incarnation et création artistique. Les artistes créent de la sorte une ambiguïté au point de laisser croire que ce qu'ils font, au-delà de l'œuvre donnée à voir, est le vrai corps du Christ. En ce sens, ils sont à l'image du prêtre qui confit le corps du Christ lors du sacrifice eucharistique.

Que l'on tienne pour valable ou non l'hypothèse de Linda Seidel, celle-ci attire l'attention sur une solution de remplacement au déchiffrement des noms que l'on découvre gravés sur les édifices, ce qui devrait tempérer l'ardeur du critique à vouloir reconnaître en chacun d'eux un artiste. Il semble en effet que le phénomène décrit par l'auteur, pour autant qu'il soit correct, ne soit pas seulement repérable à Autun. L'adoption de la réforme canoniale par les moines de Saint-Ursanne (Jura suisse), entre 1095 et 1120 , décida en effet la construction d'une église sur l'emplacement de la collégiale actuelle. À cette occasion, on transféra sans doute le tombeau du saint de l'ancienne église, située au nord du cloître, dans le chevet de la nouvelle construction. De l'ancienne structure, on remploya également des éléments architectoniques, notamment deux chapiteaux qui se font face aujourd'hui dans le collatéral sud. Dans l'ensemble du décor sculpté de l'intérieur de l'édifice actuel, seuls ces chapiteaux portent des représentations à figure humaine. Attribués au XI ${ }^{\mathrm{e}}$ siècle, ils ont été placés à cet endroit par le maître d'œuvre du XII ${ }^{e}$ siècle " qui [en] fit refaire les astragales mais laissa subsister les tailloirs ». Ceci est d'importance, car sur les tailloirs on peut lire 
URSICINUS et BURCHINUS. Dans le premier, on reconnaîtra aisément le saint dont on vénère les restes à cet endroit : Ursanne ou Ursicin; quant au second, l'incertitude est entière. On a proposé sans grande conviction d'y reconnaître un sculpteur. Peut-être serait-il plus sage de considérer que Burchinus a joué un rôle important dans la translation des reliques de saint Ursanne au cours du XII ${ }^{\mathrm{e}}$ siècle, ou, ce qui parait plus vraisemblable, à quelque occasion dans une période plus reculée. Ceci permettrait d'expliquer pourquoi, au XII ${ }^{\mathrm{e}}$ siècle, on ait voulu conserver ensemble le souvenir du saint et celui de Burchinus. Cela reste de l'ordre de l'hypothèse. Pourtant, si le phénomène décrit par Linda Seidel est correct, il se pourrait que l'on soit en face d'une réponse de même ordre à Saint-Ursanne, mais dans des proportions plus modestes et moins spectaculaires ${ }^{10}$.

L'originalité du travail de Linda Seidel tient sans aucun doute au refus de considérer Saint-Lazare comme l'œuvre d'un sculpteur génial, pour y voir la volonté d'une entreprise collective dont le résultat témoigne de l'Antiquité et du pouvoir des institutions locales que représentent l'évêque, le clergé et le duc (p.159). La démonstration emprunte des chemins tortueux (Saint-Lazare comme succédané des lieux saints où effectuer un pèlerinage convainc à peine), mais par un mouvement de retours successifs, l'auteur reprend sans cesse et précise les différents arguments avancés à mesure qu'elle progresse dans l'analyse. Ce mouvement, qui rend parfois la lecture difficile, donne au récit une structure enchâssée. Il en ressort une impression entêtante : la démonstration semble elle-même le produit d'un phénomène qu'elle est censée expliquer. Le sens surgit dès l'instant où l'on entreprend de réécrire le passé. À Autun, cette réécriture du passé (à vrai dire, il s'agit plutôt d'une écriture) ne s'inscrit pas sur le parchemin, mais dans la construction d'un édifice. Mais l'on peut se demander si l'auteur n'est pas elle-même engagée sur le même chemin? Dans sa préface, Linda Seidel rappelle brièvement combien, par une fin d'après-midi de juillet, elle fut surprise de voir toute la partie droite de la nef de Saint-Lazare baignée d'une "golden light of a setting summer sun » (p. XIV). Pourquoi la lumière n'entrait-elle pas à travers le mur occidental de l'édifice, comme on l'attendrait d'une église correctement orientée? La soudaine visibilité théâtrale des reliefs ainsi mis en valeur la paralysa dans un premier temps, dit-elle, avant de déterminer le cours de toute l'enquête. Cette expérience liminale évoque, au-delà de l'extase de Kingsley Porter à Coutances, justement mentionnée par l'auteur, un épisode qui se déroula il y a plus de trois cents ans à Rome. Athanase Kircher, "mû par un instinct divin», trouve dans l'église d'Eustache une précieuse inscription qui se dérobait jusqu'alors à ses yeux : «Comme je veillais encore, occupé à cette recherche, un rayon de soleil passant par la fenêtre principale illumina une voûte entre des colonnes et fit apparaître ce texte, pour la joie et l'admiration de tous, dans les ombres que la lumière creusait dans les lettres gravées. ${ }^{11}$ Nos deux auteurs ne se rejoignent-ils pas dans cette quête érudite, où la découverte semble le fruit de l'occasion plus que du hasard? Ne découvrent-ils pas ensemble, pour reprendre encore Alain Boureau, un trésor sacré qui s'offre «à qui s'abandonne à la grâce »? L'arrivée à Autun la veille du 14 juillet 1989 et la soudaine illumination de Linda Seidel s'apparentent volontiers à la promenade érudite et à la découverte du trésor sacré d'Athanase Kircher, laissant croire que l'histoire ne peut produire de sens que dans la réécriture.

Contrairement à Linda Seidel, Jean Wirth ne cherche pas à épuiser les significations d'une œuvre particulière dans son dernier ouvrage. L'étude monographique 
s'attachant, par définition, à un objet limité, elle ne peut répondre à tout un ensemble de questions qui demandent une vision plus panoramique. Jean Wirth soulève ces questions (définition et conceptions de l'image, vocabulaire de la description, rapport de l'image au nom, chronologie et évolution stylistique) et propose d'y répondre dans L'Image à l'époque romane. La densité de cet ouvrage est telle qu'il appelle des remarques ponctuelles ou de détail, à preuve deux comptes rendus récents qui confinent à la querelle ${ }^{12}$. Rendre compte du livre de Jean Wirth n'est en effet pas chose aisée tant il fourmille d'idées originales et donne matière à réflexion ; on s'en acquittera tant bien que mal en développant plus en détail un aspect concernant l'image de l'artiste médiéval, la question des « bonnes œuvres».

"L'image à l'époque romane »

Depuis la parution de l'Art religieux du XII siècle en France d'Émile Mâle (1922), entreprendre l'étude de l'image à l'époque romane relève d'une ambition très généreuse qui ne saurait être possible, comme le reconnaît Jean Wirth, qu'à la seule condition d'offrir "une meilleure définition des courants idéologiques et [une] chronologie plus exacte [des œuvres]» (p.12). Si l'ouvrage ne prétend pas donner réponse à tout, les deux objectifs formulés en préambule par l'auteur sont le signe d'une grande rigueur méthodologique, qu'il faut souligner. Contrairement à beaucoup, Jean Wirth se refuse en effet d'abandonner l'étude iconographique aux seuls historiens. Parmi ces derniers, quelques-uns considèrent l'image médiévale comme un document qu'ils n'osent (ou ne savent) soumettre à la critique interne, au risque d'entretenir des contresens, et se contentent d'une datation approximative ou font même l'économie de la chronologie des œuvres étudiées. Si celle-ci est «irruptive» (p. 451), et semble par conséquent rebelle à tout ordonnancement régulier, il n'en demeure pas moins qu'une mise en contexte s'avère essentielle, sans perdre de vue pour autant le cadre général. $\mathrm{Au}$ cœur de la démonstration de Jean Wirth, la réforme grégorienne occupe certes une place prépondérante, mais ce n'est pas là le seul courant idéologique qui est étudié. En familier des textes théologiques, liturgiques et dévotionnels, l'auteur situe les images dans le contexte des idées contemporaines, en tenant compte de ce qui fait leur spécificité. Pour limiter les distorsions d'une étude générale, la synthèse est ainsi rythmée par des chapitres ou des parties de chapitres qui traitent un programme particulier dans le détail : les chapiteaux de Mozat (p. 154-169) ou le plafond peint de Saint-Martin de Zillis (p. 333-361), par exemple. Il faut souligner ici que les exemples choisis par l'auteur pour ménager des pauses dans le développement général posent tous, à des degrés divers, des problèmes sur lesquels achoppent les historiens de l'art depuis fort longtemps. Ces problèmes sont essentiellement d'ordre chronologique, d'où découle tout le reste, que l'on a trop souvent eu tendance à résoudre en termes "patriotiques» : seulement, la question des origines de l'art roman (faut-il en situer la naissance en Bourgogne ou dans le Sud-Ouest de la France ?) ou celle de la prééminence des édifices réputés majeurs ne font aujourd'hui plus recette. Jean Wirth propose de dépasser ces querelles somme toute provinciales en s'attachant à l'image seule, et ce de façon singulière. En plaçant d'emblée son entreprise sous l'ombre d'Émile Mâle, l'auteur prend par ailleurs le risque de voir sa démonstration lue à travers le souvenir que chacun s'est forgé de ce pionnier des études iconographiques. Si l'on laisse de côté cette provocation initiale (qui n'a pas manqué de soulever les critiques de certains), on se rendra compte rapidement de la différence des deux démarches. Là où É. Mâle tendait à une vision globale sinon globalisante du phénomène visuel médiéval, J. Wirth propose une somme de courtes analyses réunies en une synthèse qui ne prétend jamais 
à l'exhaustif; là où É. Mâle donnait l'impression de rechercher le texte qui justifierait toute image, J. Wirth s'intéresse à l'image seule pour en débusquer les singularités, les étrangetés, les déviations. Pour expliquer ensuite ces «discrépances ", l'auteur mène son analyse iconographique en s'appuyant sur de multiples sources historiques et textuelles. Bien que la bibliographie ne le reflète pas ouvertement, les savantes constructions de l'auteur s'élèvent sur une solide structure faite de sources, qu'il maîtrise à la perfection, et de littérature secondaire. Car il ne faut pas se méprendre sur les buts visés par cette étude : il ne s'agit pas tant de rendre compte de l'image romane ou, pour le mettre dans les termes d'Émile Mâle, de procéder à un déchiffrement iconographique, que de parler d'image. Concrètement, l'auteur cherche à déterminer les ressorts et les pratiques de la représentation, qu'il soupçonne être organisés en système. Partant du principe que la pensée s'offre elle-même comme système, le même qui sous-tend les représentations, l'auteur entend montrer comment ils se chevauchent. On pourrait soupçonner ici l'existence d'un diallèle: les images « indicielles » qui permettent de débusquer le système sont convoquées par la suite pour en prouver justement l'existence. Mais cette circularité n'est qu'apparente: le raisonnement s'appuie sur des principes premiers, établis en partie dans un précédent ouvrage de l'auteur ${ }^{13}$, et l'on ne voit pas d'où pourraient provenir les indices tant recherchés sinon des images elles-mêmes.

Parler de l'image à l'époque romane suppose donc avant tout qu'on la définisse. La première partie de l'étude, qui couvre une période allant d'Augustin au synode d'Arras de 1025 , est justement consacrée à la question de l'héritage de la période carolingienne. L'auteur montre comment l'image, considérée comme un ornement par les Carolingiens, devient le signe tangible d'un optimisme croissant sur les possibilités de représenter le spirituel. $\mathrm{Au} \mathrm{xI}{ }^{\mathrm{e}}$ siècle, ceci aboutit à une conception de l'image comme forme la plus commune de la présence du sacré et amène donc à rendre visibles Dieu, le Christ et ses saints - Jean-Claude Bonne parlerait ici de "médiété " de l'image médiévale. Cette période est un moment charnière dans l'analyse de Jean Wirth, puisqu'elle consacre une crise du système iconographique. C'est alors que l'on assiste au déclin du narratif qui se réduit à l'image (au sens médiéval du terme) ou disparaît au profit de l'ornemental (et l'on peut suivre le développement de formes non figuratives, de lettres ornées, qui conduit à l'éclosion de la split representation, ou figura geminata dans le langage médiéval). Parallèlement, la période se caractérise par une attitude plus compréhensive envers les comportements religieux toujours empreints d'un certain "paganisme", que l'Église absorbe (voir les pages consacrées à la femme de l'Apocalypse et à la sirène romane, p. 134-154). L'auteur note avec raison l'éclatement des structures de l'image au $\mathrm{XI}^{\mathrm{e}}$ siècle, notamment la réorganisation des rapports qu'elle entretient avec le fond et le cadre, qui mène, sur le plan formel, à la création du champ, et prélude à la crise, plus importante, de la ressemblance qui conduit au mépris sinon à la diabolisation de la chair. Si l'image est capable de représenter l'invisible au $\mathrm{XI}^{\mathrm{e}}$ siècle, $\mathrm{c}^{\prime}$ est un mouvement inverse qui se fait jour autour de 1100. La réhabilitation de la nature et de l'homme a pour conséquence une réorganisation des rapports de la chair et de l'esprit, pensés non plus dans une relation d'opposition mais de subordination de la première au second (cela se remarque entre autres, dit Jean Wirth, à un certain assouplissement du style). Les religieux ascétiques se tournent alors vers l'imagination, conçue comme une médiation entre l'ici-bas et ce vers quoi l'on tend, et les images "pour organiser leur vie affective». Au terme de l'évolution, Marie personnifie à la fois la communauté et le clergé pour devenir la figure du rôle 
médiateur de l'Ecclesia. Elle occupe désormais la place centrale du système. L'évolution de l'iconographie mariale, d'une déesse lunaire à l'allégorie ecclésiale particulière, signale ainsi «l'irrésistible ascension de la Vierge » (p.441) qui caractérise l'époque romane.

L'auteur note avec raison que le $\mathrm{xI}^{\mathrm{e}}$ siècle tend à donner au spirituel un caractère concret, ce qui amène à penser Dieu comme res au moment où la seconde querelle eucharistique trouve une fin provisoire au concile de 1079. L'aboutissement d'une nouvelle conception de l'eucharistie et la réforme de l'institution ecclésiastique débouchent sur la consolidation du pouvoir pontifical et la sacralisation du statut du prêtre, dont profiteront les artistes. Les conséquences sur la fabrication de l'image, pas seulement celle de Dieu, sont en effet importantes: le rejet de la chair conduit à valoriser les œuvres humaines, que l'on retrouve opposées à celles de la luxure et de l'avarice dans de nombreux programmes sculptés par exemple. L'importance des œuvres sans lesquelles la foi n'est rien (Jc 2,$14 ; 17)$, une importance bien mise en évidence par Jean Wirth (p. $303 \mathrm{sq}$.), a pour conséquence entre autres la diffusion de la signature et de la représentation de l'artiste. L'œuvre artistique acquiert ainsi une valeur religieuse, comme le remarque justement l'auteur en convoquant l'exemple de Guillelmus à la façade de San Zeno à Vérone (p. 317). Malheureusement, il s'abstient de préciser que les signatures de ce type s'apparentent aux nombreux colophons dans lesquels le scribe appelle le lecteur à prier pour son âme en échange du travail accompli. Aux alentours de l'an mil déjà, le fondeur Berengerus invitait le lecteur passant à en faire de même sur la porte de la cathédrale de Mayence : BERENGERUS HUIUS OPERIS ARTIFEX LECTOR UT P[RO] EO D[EU]M ROGES POSTULAT SUPPLEX (voir également les exemples cités plus haut). Mais si Jean Wirth a raison de souligner la forte valorisation des œuvres à la suite de la réforme grégorienne, le phénomène se repère avant, et sans doute serait-il nécessaire de situer mieux les œuvres romanes dans cette ascendance. La valorisation de l'œuvre créée (comme de l'œuvre offerte) permet l'assimilation de l'artiste au Créateur et du Créateur à un artiste, et signale la réhabilitation de la nature dès la seconde décennie du XII ${ }^{\mathrm{e}}$ siècle environ. Deux exemples, cités par Jean Wirth, sont explicites : sur la façade sud de San Zeno à Vérone, Nicolaus se compare ouvertement à Dieu créateur d'Adam, tandis qu'au tympan de la Sainte-Trinité d'Autry-Issards, Natalis se glisse dans une trinité créatrice bien particulière, au point de se substituer à l'Esprit Saint.

Le travail artistique comme « bonne œuvre »

18 Si l'on suit l'iconographie du sculpteur au travail au XII siècle, on se rendra compte que deux types de représentations co-existent. Dans les premières, le sculpteur est occupé à dégrossir un bloc : c'est le cas à la porte de bronze de San Zeno de Vérone (vers 1138) ou sur un chapiteau de la galerie occidentale du cloître de la cathédrale de Gerona (entre 1179 et 1196) ; sur un chapiteau de Saint-Servais de Maastricht (vers 1160-1180) encore, les lapicides sont clairement désignés comme des operarii œuvrant sur une pierre (lapis). Sans doute peut-on rapprocher ces représentations des marques de tâcherons repérables dans de nombreux édifices romans, dont quelques-unes s'apparentent parfois aux authentiques signatures de sculpteurs. C'est notamment le cas du lapicide Ugo, actif entre 1125 et 1160 environ dans la Provence rhodanienne, qui se contente d'ordinaire de graver son nom, mais le fait suivre d'un me fecit à la chapelle de Saint-Blaise-de-la-Martre. Dans le second cas, et pour signaler sans ambiguïté qu'il n'est ni un lapicide ni un maçon, le sculpteur se montre taillant un chapiteau, le plus 
souvent corinthien. C'est le cas dans l'archivolte de la Porta dei Principi du dôme de Modène (vers 1120-1140), d'un chapiteau de La Daurade à Toulouse, daté du dernier quart $\mathrm{du} \mathrm{XII}^{\mathrm{e}}$ siècle (où l'on devine par ailleurs que le sculpteur porte de ravissantes chaussures décorées de broderies, peu pratiques pour travailler dans l'atelier poussiéreux d'un sculpteur), ou d'un chapiteau du cloître de San Cugat del Vallès près de Barcelone (fin XII ${ }^{e}$ siècle), signé par Arnaud Catell ${ }^{14}$. La signature particulièrement visible d'Unbertus sur le tailloir d'un chapiteau corinthien du porche de Saint-Benoîtsur-Loire (vers 1030) manifeste la fierté légitime du sculpteur. L'emphase portée sur le chapiteau appelle les exemples de donateurs contemporains. À Sainte-Marie de Maastricht (dernier quart du XII ${ }^{\mathrm{e}}$ siècle), Heimo en offre un à la Vierge, tandis que Stefanus l'offre à un ange sur un chapiteau du chœur de Notre-Dame-du-Port à Clermont-Ferrand (vers 1100). Le phénomène se repère déjà au milieu du $\mathrm{IX}^{\mathrm{e}}$ siècle sur l'autel d'or de Saint-Ambroise à Milan où le portrait de Volvinus, couronné par le saint, fait pendant à celui du donateur Angilbert. Si ces exemples insistent sur l'œuvre artistique comme œuvre, à savoir en tant que terme d'un échange dans l'économie générale du salut, échange auquel les artistes participent de plus en plus activement, il faut attendre le milieu du XII ${ }^{\mathrm{e}}$ siècle toutefois pour voir un artiste inscrire ouvertement son activité dans la thématique des bonnes œuvres.

Le portail occidental du prieuré d'Autry-Issards (entre 1140 et 1150), dédié à la SainteTrinité, développe un programme intéressant, au sein duquel se mêlent l'iconographie et l'épigraphie. Si l'on veut dégager le programme iconographique du tympan, il faut s'appuyer entre autres sur la présence de l'archange Raphaël, identifié par une inscription; cette présence explicite est assez rare à cette époque pour justifier une attention soutenue. Ange médecin ou ange gardien, Raphaël manifeste ici la bienveillance divine portée à celui qui accomplit de bonnes œuvres. Le tympan d'AutryIssards met en scène les œuvres, notamment les bona opera qu'il faut accomplir ici-bas en vue de la récompense éternelle. L'iconographie et l'épigraphe qui court au linteau les énumèrent tout en les associant : Création divine et Incarnation, qui sont les œuvres de la Trinité ; les œuvres de justice, dont témoignent les deux archanges ; et l'œuvre du sculpteur Natalis qui signe au tympan. L'épigraphe dit en effet : + CUNCTA DEUS FECI HOMO FACTUS CUNCTA REFECI+NATALIS ME FEC[IT $]^{15}$. Il est donc légitime de formuler l'hypothèse selon laquelle il existe une relation entre les trois «œuvres" (et leurs auteurs sans doute) décrites au tympan, à savoir Création divine, Incarnation et création artistique (considérée comme une bonne œuvre). La première relation est évidente et il n'est pas nécessaire de s'y attarder : l'Incarnation étant conçue comme une re-création, les deux sont placées dans une relation de continuité ou d'égalité. Par contre, il faut analyser soigneusement les deux relations restantes: entre Incarnation et création artistique d'une part, et entre Création divine et création artistique d'autre part. L'épigraphe place à la suite l'un de l'autre номо ғAстUs (le Christ) et le sculpteur qui le fait; ceci constitue un indice qui permet d'entrevoir une explication. Natalis n'est pas le seul à affirmer en effet qu'il «fait » le Christ (Jean Wirth signale ainsi Rotbertus à ClermontFerrand et Gofridus à Chauvigny, p. 316), à l'image du prêtre qui confit le vrai corps du Christ lors de la consécration eucharistique. La seconde relation met en parallèle Création divine et création artistique : les thèmes croisés du Deus artifex et du divin artiste jouent sans doute ici un rôle, qu'il faut expliquer, mais il convient également de s'attacher à la façon dont Dieu crée (par la parole ou par le geste, ce qui se remarque très bien dans les images) et ses conséquences sur l'artiste médiéval. Ainsi, l'exemple 
d'Autry-Issards engage-t-il la discussion sur ce qui véritablement créé; et l'on peut soupçonner qu'il s'agit aussi, au-delà de cette église particulière, de l'ecclesia spiritualis ou de la Jérusalem céleste. Natalis signale ainsi que les bonnes œuvres accomplies icibas s'inscrivent dans l'au-delà. Si l'on se tourne une dernière fois vers l'épigraphe d'Autun, on pourra dès lors comprendre le pronom hoc comme renvoyant précisément à cette église spirituelle dont Saint-Lazare serait l'image terrestre.

Même s'il exige de son lecteur une attention soutenue, L'Image à l'époque romane est un manuel qu'il faudra consulter souvent, parce qu'il offre une excellente introduction aux problématiques romanes et qu'il y répond par des idées originales. En ce sens, on regrettera peut-être l'absence d'un index thématique, à peine compensée par une table des matières pourtant très détaillée. Et si l'on peut relever ça et là des erreurs, elles sont imputables au caractère synthétique de l'ouvrage ; elles ne devraient aucunement servir de prétexte à le rejeter dans son entier, car ce serait manquer ce qui en fait la richesse : l'attention exclusive à l'image, à sa fabrication, sa définition, ses fonctions et ses usages, que l'auteur suit du $\mathrm{IX}^{\mathrm{e}}$ jusqu'au milieu du XII ${ }^{\mathrm{e}}$ siècle environ, qui fonde ensuite, et ensuite seulement, une analyse génétique de l'iconographie. Alors que Linda Seidel propose un système fermé, Jean Wirth offre à la lecture un système ouvert ; c'est au lecteur de l'animer désormais.

\section{NOTES}

1. L. SEIDEL, Legends in Limestone. Lazarus, Gislebertus, and the Cathedral of Autun, Chicago, The University of Chicago Press, 1999.

2. J. Wirth, L'Image à l'époque romane, Paris, Éditions du Cerf, 1999.

3. A. Legner, «Illustres manus », dans Ornamenta Ecclesiae. Kunst und Künstler der Romanik, 3 vol., Cologne 1985, I, p. 187-230 ; P. C. CLAUSSEN, Magistri doctissimi romani. Die römischen Marmorkünstler des Mittelalters, Stuttgart, 1987 ; P. Skubiszewski, «L'intellectuel et l'artiste à l'époque romane », dans Le Travail au Moyen Âge. Une approche interdisciplinaire, Louvain-la-Neuve, 1990, p. 263-313 ; P. C. CLAUSSEN, "Nachrichten von den Antipoden oder der mittelalterliche Künstler über sich selbst », dans Der Künstler über sich und in seinem Werk, Weinheim, 1992, p. 19-54. Parmi les ouvrages les plus récents, il convient de signaler encore A. Erlande-Brandenburg, De pierre, d'or et de feu. La création artistique au Moyen Âge, IV ${ }^{e}$-XIII ${ }^{e}$ S., Paris, 1999.

4. V. W. EGBeRT, The Medieval Artist at Work, Princeton, 1963 et A. MARTINDALE, The Rise of the Artist in the Middle Ages and Early Renaissance, Londres, 1972.

5. Le buticularius est l'un des "ministres » présents dans l'entourage de l'évêque ou du prince, comme le sont aussi le senescalcus, le camerarius, le coquinarius, le stabularius, etc., et occupe une charge élevée au sein de l'équipage de cour. En ce sens, échanson me semble plus précis que cellar master. À aucun moment, l'auteur n'envisage de reconnaître un seul Gislebertus qui, de buticularius vers 1120 , serait capellanus peu avant 1150 ; rien ne s'oppose en effet à ce que ce soit ce même Gislebertus qui signe au tympan d'Autun. Le moine de Saint-Gall Tuotilo (ix ${ }^{e} s$.), outre son expertise en divers 
arts, occupa plusieurs fonctions au sein de son monastère, dont celle de cellérier. Odorannus de Sens (première moitié $\mathrm{du} \mathrm{XI}^{\mathrm{e}} \mathrm{s}$.) est également un moine aux talents multiples : architecte, orfèvre, musicien peut-être, il maîtrisait les arts du trivium comme ceux du quadrivium et composa entre autres plusieurs oraisons et un rite pour la commémoration des défunts. Le cas d'Arnaud Catell est rappelé plus bas à la note 14 . 6. R. Favreau, Épigraphie médiévale (L'atelier du médiéviste, 5), Turnhout, 1997, p. 32. 7. Au début du XIII ${ }^{\mathrm{e}}$ s., ce même sculpteur signe le tympan occidental d'un ermitage situé à Puilampa, dans la province de Saragosse.

8. Il semble toutefois que la plaque ne soit pas solidaire du tympan et qu'elle ait été insérée plus tard dans sa bordure inférieure, probablement en 1812.

9. Sur la réception de Priscien, voir R. W. HUNT, The History of Grammar in the Middle Ages. Collected Papers, Amsterdam, 1980. Au début du XII ${ }^{\mathrm{e}}$ s., la glose sur Priscien précise : "pronomen vero significat substantiam sine qualitate".

10. Au moment de la transformation du monastère en collégiale, les archives disparurent et avec elles sans doute toute information qui aurait pu être utile pour identifier ce mystérieux mais, semble-t-il, important personnage. Voir C. Lapaire, Les Constructions religieuses de Saint-Ursanne et leurs relations avec les monuments voisins, VIIXIII ${ }^{e}$ s., Porrentruy, 1960 (cit. p. 39).

11. L'épisode est librement inspiré d'A. Boureau, L'Événement sans fin. Récit et christianisme au Moyen Âge, Paris, 1993, p. 112.

12. Ses erreurs ont été rassemblées par Y. Christe dans un compte rendu paru dans le Bulletin Monumental (CLVIII, 2000), auquel J. Wirth a répondu (Bulletin Monumental, CLIX, 2001, p. 205-206), et par R. A. Maxwell (Art Bulletin, LXXXIII, 2001, p. 757-762), à qui on laisse bien volontiers le soin de croire que, de ce côté-ci de l'Atlantique, l'on découvre à peine les ouvrages d'Erwin Panofsky!

13. J. Wirth, L'Image médiévale. Naissance et développements ( $\mathrm{VI}^{e}-\mathrm{XV}{ }^{e} \mathrm{~s}$.), Paris, 1989.

14. En 1206 et 1207, Arnaud Catell signe comme témoin des chartes en rapport avec le monastère qu'il a édifié. Son exemple n'est pas sans rappeler celui de (Petrus ?) Brunus qui signe deux des apôtres de la façade de Saint-Gilles-du-Gard (brunus me fecit) et intervient comme témoin signataire de plusieurs actes notariés relatifs à Saint-Gilles et à Nîmes en 1171 et 1186.

15. La transcription est erronée chez Jean Wirth, p. 378.

\section{AUTEUR}

\section{PIERRE ALAIN MARIAUX}

Institut d'Histoire de l'art, Espace Louis-Agassiz 1, Université de Neuchâtel, CH-2000 Neuchâtel 